\title{
Long non-coding RNA ANRIL promotes the proliferation, migration and invasion of human osteosarcoma cells
}

\author{
SHUO CHENG, TAO HUANG, PENGCHENG LI, WEI ZHANG, ZHIHANG WANG and YAO CHEN
}

Department of Orthopedics, The First Affiliated Hospital of China Medical University, Shenyang, Liaoning 110001, P.R. China

Received November 10, 2016; Accepted July 14, 2017

DOI: 10.3892/etm.2017.5123

\begin{abstract}
The long antisense non-coding RNA ANRIL is $3.8 \mathrm{~kb}$ in length and serves an important role in the tumorigenesis and progression of a number of malignancies. However, its function in human osteosarcoma remains unknown. The present study investigated the role of ANRIL in human osteosarcoma cell lines and clinical tumor samples. The expression of ANRIL was analyzed in 19 osteosarcoma and paired adjacent non-cancerous tissues using reverse transcription-quantitative polymerase chain reaction. Knockdown of ANRIL expression using lentivirus-mediated small interfering RNA was performed to investigate the role of ANRIL in tumor proliferation and metastasis using MTT, colony formation and transwell assays. The results demonstrated that ANRIL expression was upregulated in osteosarcoma tissues when compared with ANCT samples. In addition, knockdown of ANRIL expression in vitro reduced cell proliferation and invasion. These results indicated that ANRIL is upregulated in osteosarcoma tissues, and may promote the proliferation and metastasis of osteosarcoma cells. Therefore, ANRIL may serve as a novel biomarker and target of novel therapies for the treatment of patients with osteosarcoma.
\end{abstract}

\section{Introduction}

Osteosarcoma is a tumor of mesenchymal origin that accounts for $20 \%$ of all primary bone cancers. Osteosarcoma develops primarily among children and adolescents and demonstrates a low 5-year survival rate, a high amputation rate and a poor post-operative function recovery rate $(1,2)$. Long non-coding RNAs (lncRNAs) are $>200$ nucleotides in length and are non-protein-coding capacity transcripts. Previous studies have demonstrated that lncRNAs regulate the expression of genes at different levels through a variety of mechanisms, including

Correspondence to: Professor Tao Huang, Department of Orthopedics, The First Affiliated Hospital of China Medical University, 155 North Nanjing Street, Shenyang, Liaoning 110001, P.R. China

E-mail: huangtao_cmu@126.com

Key words: ANRIL, long non-coding RNA, osteosarcoma chromatin modification, transcription, splicing, translation and post-transcriptional regulation $(3,4)$. It has been demonstrated that IncRNAs also serve important roles in the biological processes of cancer cells, including cell proliferation, invasion, differentiation and apoptosis (5-7). Deregulated lncRNA expression has been observed in various cancers, which suggests that lncRNAs may serve a vital regulatory function in tumorigenesis and cancer progression $(8,9)$.

The ANRIL lncRNA is transcribed as a $3.8 \mathrm{~kb}$ mRNA in the opposite orientation of INK4b-ARF-INK4a gene cluster, which has been identified as a genetic susceptibility locus associated with coronary disease, intracranial aneurysm, type 2 diabetes and various types of cancer $(10,11)$. In addition, ANRIL activates polycomb repressive complexes (PRC) 1 and PRC2, to regulate the expression of INK4bARF-INK4a (12,13). Zhang et al (14) demonstrated that ANRIL expression was increased in gastric cancer tissues, and was associated with tumor size and tumor, node, metastasis (TNM) stage. Further studies have demonstrated that ANRIL knockdown significantly inhibits the proliferation and invasion of cancer cells in vitro and in vivo (14). Huang et al (15) identified that the expression of IncRNA ANRIL is increased in hepatocellular carcinoma, and demonstrated that it serves a role in tumor proliferation and metastasis. The results of these studies therefore suggest that dysregulation of ANRIL may be a contributing factor in human cancer progression. However, the functional role and underlying mechanisms of action of ANRIL in osteosarcoma remain unknown.

The present study investigated the biological functions of the ANRIL IncRNA in osteosarcoma development by examining the expression of ANRIL in osteosarcoma tissues. In vitro assays were subsequently performed to identify the biological functions of ANRIL in an osteosarcoma cell line.

\section{Materials and methods}

Patients and tissue samples. Osteosarcoma tissues and their adjacent non-cancerous tissues (ANCT) were obtained from 19 patients (10 males and 9 females; $15-35$ years) pathologically diagnosed with osteosarcoma that underwent resection of osteosarcoma at the First Affiliated Hospital of China Medical University (Shenyang, China) between July 2010 and July 2014. None of the patients received therapy prior to surgery. Patients at stage IIB/III were included, and patients with any other primary disease were excluded. All tissues 
were immediately stored in liquid nitrogen and maintained at $-80^{\circ} \mathrm{C}$. The associated clinical data (including sex, age, TNM stage) was collected from each of the patient's medical records. The current study was approved by the Ethics Committee of the First Affiliated Hospital of China Medical University, and informed consent was obtained from all participants.

Cell culture. The human osteosarcoma cell line U2OS (Shanghai Gefan Biotechnology, Shanghai, China) was cultured in RPMI-1640 medium (Gibco; Thermo Fisher Scientific Inc., Waltham, MA, USA) supplemented with $10 \%$ fetal bovine serum (FBS; PAN-Biotech GmbH, Aidenbach, Germany) and $5 \%$ horse serum (Gibco; Thermo Fisher Scientific, Inc.) at $37^{\circ} \mathrm{C}$ in $5 \% \mathrm{CO}_{2}$.

Lentivirus-mediated RNA interference. U2OS cells were transfected with lentivirus core vector (hU6-MCSCMV-RFP) carrying shRNA targeting human ANRIL (si-ANRIL) and non-targeting control (si-NC), 5'-TTCTC CGAACGTGTCACGT-3' (GeneChem Co., Ltd., Shanghai, China). The sequence of the shRNA target for ANRIL was 5'-GGUCAUCUCAUUGCUCUAU-3' (GeneChem Co., Ltd.), which has been identified as an effective interference target sequence of ANRIL by Kotake et al (12). U2OS cells were infected at a multiplicity of infection of 20, and incubated for $72 \mathrm{~h}$ prior to performing the subsequent assays.

Cell proliferation assays. Cell viability was measured using an MTT kit (Nanjing KeyGen Biotech Co., Ltd., Jiangsu, China) according to the manufacturer's instructions. A total of 3,000 transfected cells/well were seeded in 96-well plates and incubated at $37^{\circ} \mathrm{C}$ in $5 \% \mathrm{CO}_{2}$ for 1 day. Then, $50 \mathrm{ml} \mathrm{MTT}$ solution was added into the medium for $4 \mathrm{~h}$ incubation at $37^{\circ} \mathrm{C}$ in $5 \% \mathrm{CO}_{2}$, then each well was replaced with $150 \mathrm{ml}$ dimethyl sulfoxide. The absorbance of each sample was recorded at $490 \mathrm{~nm}$. Cell proliferation was analyzed at 24, 48, 72 and $96 \mathrm{~h}$.

The colony formation assay was performed by seeding transfected cells into each well of a 6 -well plate $(500$ cells/well) containing RPMI-1640 medium supplemented with $10 \%$ FBS, and incubating the cells for 2 weeks. The medium was refreshed every 4 days. Colonies were fixed with pure methanol for $15 \mathrm{~min}$ at room temperature and stained with $0.1 \%$ crystal violet in PBS for $15 \mathrm{~min}$ at room temperature. The total number of stained colonies was counted. The experiments were performed in triplicate.

Cell migration and invasion assays. At $48 \mathrm{~h}$ following transfection, U2OS cells were harvested. The migration assay was performed by seeding $1 \times 10^{5}$ transfected cells in the upper chamber of a Transwell insert (Corning Incorporated, Corning, NY, USA). The invasion assay was performed by seeding $1 \times 10^{5}$ transfected cells in the upper chamber containing RPMI-1640 medium without serum and coated with Matrigel (Corning Incorporated). The inserts were placed in the lower chamber wells of a 24-well plate containing Dulbecco's modified Eagle's medium supplemented with $10 \%$ FBS. Following $24 \mathrm{~h}$ incubation at $37^{\circ} \mathrm{C}$ in $5 \% \mathrm{CO}_{2}$, cells remaining on the upper membrane were removed by scraping with a cotton swab. The migrated or invaded cells on the lower membrane were fixed with pure methanol for $10 \mathrm{~min}$ at room temperature and stained with $0.1 \%$ crystal violet for $15 \mathrm{~min}$ at room temperature. Cell numbers were counted under a Leica DMi8 inverted microscope (Leica Microsystems $\mathrm{GmbH}$, Wetzlar, Germany) at magnification, x200. Experiments were independently repeated in triplicate.

$R N A$ extraction and reverse transcription-quantitative polymerase chain reaction $(R T-q P C R)$. Total RNA was extracted from tissues or cells using TRIzol ${ }^{\circledR}$ reagent (Invitrogen; Thermo Fisher Scientific Inc.). cDNA synthesis was performed using the PrimeScript RT Reagent kit with gDNA Eraser (cat. no. RR047A; Takara Biotechnology Co., Ltd., Dalian, China). qPCR was performed with SYBR Premix Ex Taq (Takara Biotechnology Co., Ltd.). The results were normalized to the expression of GAPDH. The sequences of specific RNA primers for ANRIL and GAPDH were as follows: ANRIL forward, 5'-CTGATTCAACAGCAGAGATCA AAGA-3' and reverse, 5'-CACACCTAACAGTGATGCTTG AAC-3'; GAPDH forward, 5'-GTCAACGGATTTGGTCTG TATT-3' and reverse, 5'-AGTCTTCTGGGTGGCAGTGAT-3'. RT-qPCR analysis and data collection were performed using an Applied Biosystems 7900 Fast Real-Time PCR system (Applied Biosystems; Thermo Fisher Scientific, Inc.). The thermocycling conditions were as follows: Initial denaturation stage $\left(95^{\circ} \mathrm{C}\right.$ for $\left.30 \mathrm{sec}\right)$; PCR reaction stage (40 cycles of $95^{\circ} \mathrm{C}$ for $5 \mathrm{sec}$ and $60^{\circ} \mathrm{C}$ for $\left.34 \mathrm{sec}\right)$; dissociation stage $\left(95^{\circ} \mathrm{C}\right.$ for $15 \mathrm{sec}, 60^{\circ} \mathrm{C}$ for $1 \mathrm{~min}$ and $95^{\circ} \mathrm{C}$ for $\left.15 \mathrm{sec}\right)$. The relative expression of ANRIL was calculated and normalized using the $2^{-\Delta \Delta \mathrm{Cq}}$ method (16).

Statistical analysis. All statistical analyses were performed using SPSS 18.0 (SPSS, Inc., Chicago, IL, USA). Significant differences between groups were estimated using a Student's t-test. $\mathrm{P}<0.05$ was determined to indicate statistically significant difference.

\section{Results}

ANRIL is upregulated in osteosarcoma tissues. RT-qPCR was performed to detect the level of ANRIL expression in 19 osteosarcoma tissues and ANCT samples. The results demonstrated that ANRIL expression was significantly upregulated in osteosarcoma tissues when compared with ANCT (P<0.05; Fig. 1).

Knockdown of ANRIL inhibits the proliferation of osteosarcoma cells. The function of ANRIL on the proliferation of osteosarcoma cells was investigated by lentivirus-mediated knockdown of ANRIL expression in U2OS cells. Transfected cells labeled with red fluorescent protein were observed under a fluorescence microscope, and the transfection efficiency was $\sim 80 \%$ (Fig. 2A and B). The RT-qPCR results demonstrated that ANRIL expression was significantly decreased in U2OS cells transfected with si-ANRIL compared with the si-NC group $(\mathrm{P}<0.05$; Fig. $2 \mathrm{C})$. The MTT assay results demonstrated that knockdown of ANRIL expression significantly decreased the proliferation of U2OS cells when compared with the si-NC group at 48, 72 and $96 \mathrm{~h}$ following transfection $(\mathrm{P}<0.05$; Fig. 3A). Similarly, the colony formation assay indicated that knockdown of ANRIL significantly decreased the number of U2OS cell colonies ( $\mathrm{P}<0.05$; Fig. 3B). 


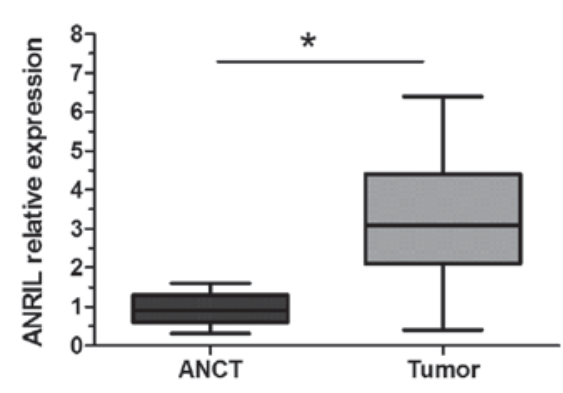

Figure 1. Relative expression of the ANRIL lncRNA in osteosarcoma tissues compared with ANCT. ANRIL expression was detected using reverse transcription-quantitative polymerase chain reaction and normalized to GAPDH expression. ${ }^{~} \mathrm{P}<0.05$. Data are presented as the mean \pm standard deviation of three independent experiments. LncRNA, long non-coding RNA; ANCT, adjacent non-cancerous tissues.
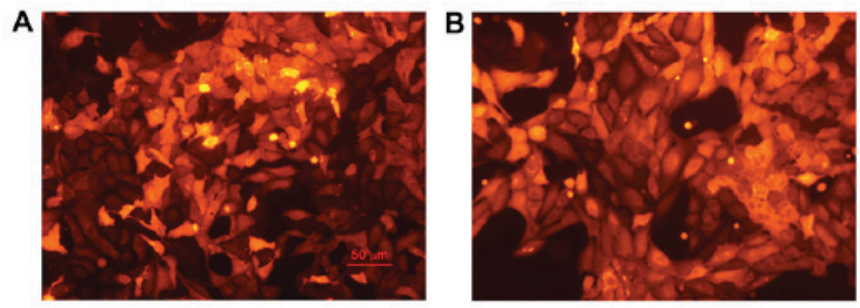

C

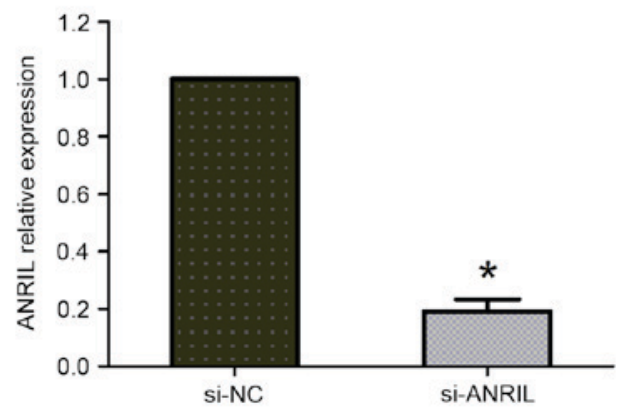

Figure 2. Relative ANRIL expression in U2OS cells following lentivirus-mediated shRNA transfection. The transfection efficiency was determined in U2OS cells transfected with (A) si-ANRIL and (B) si-NC at $72 \mathrm{~h}$ following incubation with lentiviruses at a multiplicity of infection of 20 . Transfected cells labeled with red fluorescent protein were observed under a fluorescence microscope. (C) Total RNA was extracted at $72 \mathrm{~h}$ following transfection, and relative ANRIL expression was determined using reverse transcription-quantitative polymerase chain reaction. ANRIL expression levels were normalized to that of GAPDH. Experiments were independently repeated in triplicate. Data are presented as the mean \pm standard deviation of three independent experiments. " $\mathrm{P}<0.05$. si-RNA, small interfering RNA; NC, negative control.

Downregulation of ANRIL inhibits the invasion and migration of osteosarcoma cells. The effect of knockdown of ANRIL expression on osteosarcoma cell invasion and migration was investigated using a Transwell invasion and migration assay, respectively. The results demonstrated that the invasion ability of U2OS osteosarcoma cells transfected with si-ANRIL was significantly decreased compared with the si-NC group $(\mathrm{P}<0.05$; Fig. $3 \mathrm{C}$ and $\mathrm{D})$. Similar results were observed in the migration assay, whereby U2OS cells transfected with si-ANRIL exhibited a significantly lower level of migration when compared with the si-NC group $(\mathrm{P}<0.05$; Fig. $3 \mathrm{C}$ and $\mathrm{D})$. These results suggest that ANRIL may promote osteosarcoma cell metastasis.

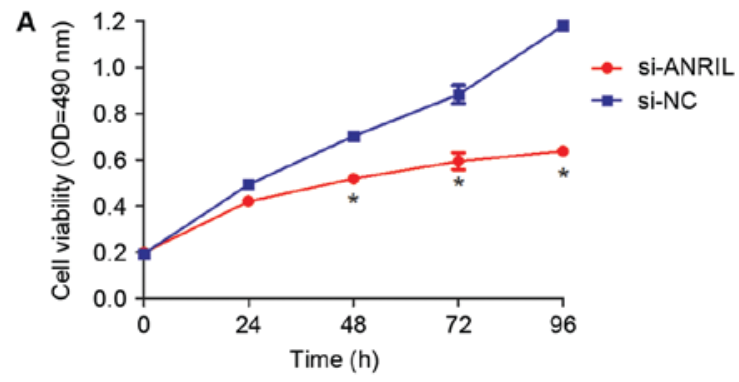

B
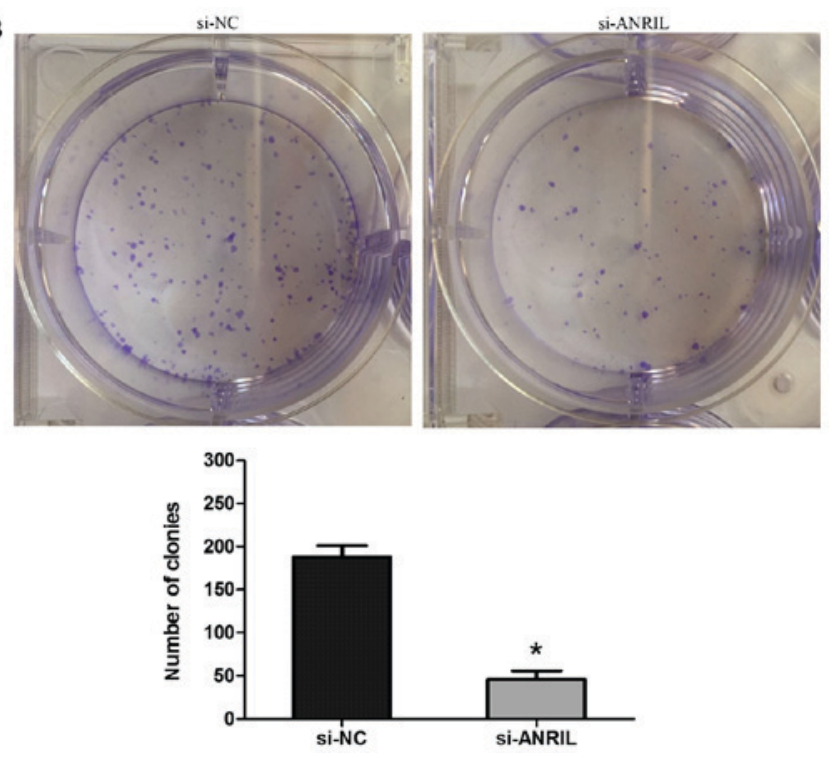

C
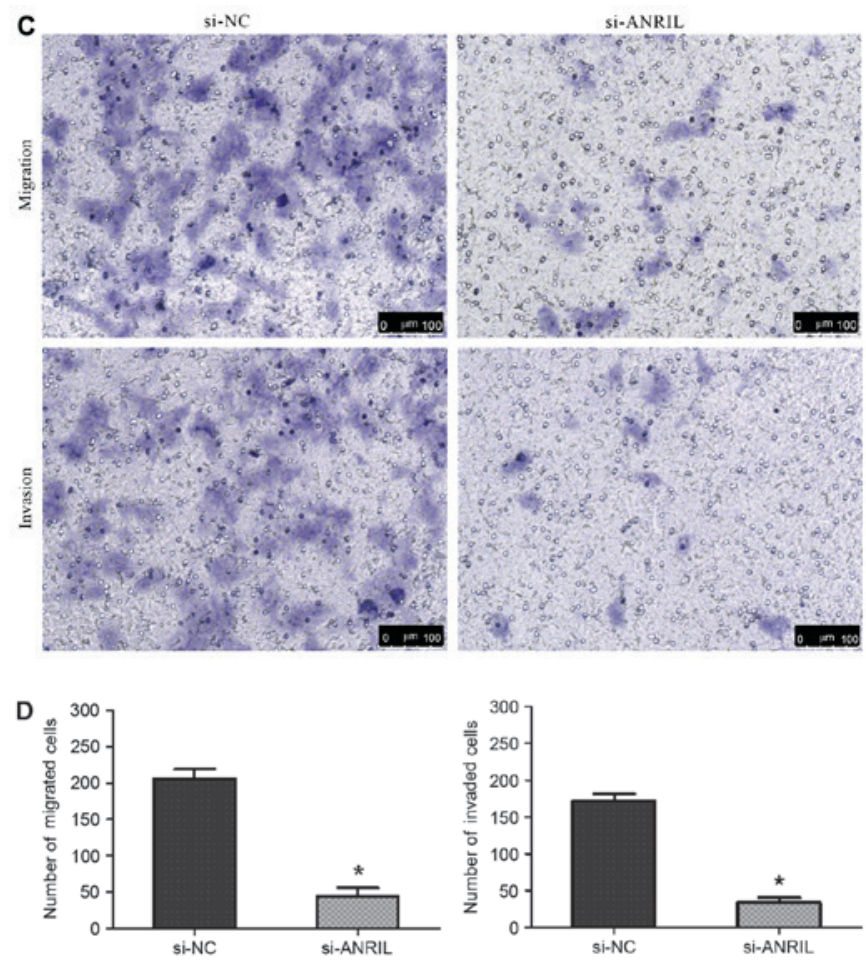

Figure 3. Downregulation of ANRIL inhibits U2OS cell proliferation, migration and invasion. (A) MTT assay indicating the proliferation of U2OS cells transfected with si-ANRIL or si-NC ( $\mathrm{P}<0.05$ si-ANRIL vs. si-NC at each time point). (B) Colony formation assay of U2OS cells transfected with si-NC or si-ANRIL. (C) Transwell migration and invasion assays indicating the migration and invasion of U2OS cells transfected with si-ANRIL or si-NC. (D) Quantification of the migration and invasion capabilities of transfected U2OS cells. Experiments were independently repeated in triplicate. Data are presented as the mean \pm standard deviation of three independent experiments. ${ }^{*} \mathrm{P}<0.05$ vs. si-NC. si-NC, small interfering RNA-negative control. 


\section{Discussion}

Up to $70 \%$ of the human genome is transcribed into RNA, and only $\sim 2 \%$ of the genome is composed of protein-encoding genes (1). Therefore, the human genome contains higher non-coding information than coding information, which subsequently leads to the expression of a greater number of non-coding RNA (ncRNA) transcripts. NcRNAs are commonly divided into small ncRNAs and lncRNAs. LncRNAs are $>200$ nucleotides in length, are non-coding and lack an open reading frame.

LncRNAs are emerging as novel gene regulators that are associated with various human diseases, including different cancers (17-19). It has been demonstrated that the dysregulation of lncRNAs may serve a role in tumorigenesis and cancer progression (3). Gupta et al (20) demonstrated that the expression of hox transcript antisense RNA (HOTAIR) lncRNA was increased in primary breast tumors, and may be a useful predictor of subsequent metastasis and mortality. In addition, growth arrest specific 5 has been identified to promote prostate cancer cell apoptosis, and as the cells acquire castrate-resistance, its expression decreases (8). Wang et al (21) demonstrated that the expression of the PlncRNA-1 lncRNA was significantly higher in human esophageal squamous cell carcinoma, and was correlated with lymph node metastasis and an advanced clinical stage. Knockdown of PlncRNA-1 expression inhibited cell proliferation and increased apoptosis in vitro (21). Previous studies have also indicated that transcription factors, c-myc and p53, activate HOTAIR transcription and regulate PVT-1 expression, respectively $(22,23)$. The results of these studies suggest that the aberrant expression of lncRNAs may be associated with the progression of multiple tumor types, and may be useful as a prognostic indicator. However, the function of ANRIL in osteosarcoma remains unknown.

The ANRIL IncRNA is transcribed as a $3.8-\mathrm{kb}$ mRNA in the opposite orientation of the INK4b-ARF-INK4a gene cluster. The INK4b-ARF-INK4a locus encodes three critical tumor suppressors, p16INK4A, p14ARF and p15INK4B. Common disease genome wide association studies have identified the ANRIL gene as a genetic susceptibility locus associated with coronary disease, intracranial aneurysm and type 2 diabetes. ANRIL interacts with p16INK4A, p14ARF and p15INK4B. When ANRIL combines with chromobox 7 (CBX7) and SUZ12 polycomb repressive complex 2 subunit (SUZ12), PRC1 and PRC2, which serve a role in chromatin modification, are activated, respectively. ANRIL promotes the interaction between SUZ12 (a component of PRC2) and the p15INK4B locus, which inhibits its expression, thus leading to an increase in cellular proliferation (12). Zhang et al (14) demonstrated that ANRIL IncRNA expression was increased in gastric cancer tissues and was associated with tumor size and TNM stage, and also have indicated that ANRIL knockdown significantly inhibits proliferation and invasion in vitro and in vivo, for instance in lung cancer cells, gastric cancer cells and liver cancer cells $(14,15,24)$. In addition, knockdown of ANRIL upregulates the expression of microRNA (miR)-99a/miR-449a in gastric cancer cell lines, SGC-7901 and BGC-823 (14). Yap et al (10) demonstrated that ANRIL and CBX7 are upregulated in prostate cancer tissues, and high expression of ANRIL and CBX7 inhibits INK4A transcription. In addition, Lin et al (24) demonstrated that ANRIL expression was increased in human non-small cell lung cancer, and the aberrant expression of ANRIL was correlated with tumor size and TNM stage. These results indicate that the dysregulation of ANRIL may serve a role in the progression of a variety of human cancers. However, the functional role and underlying mechanism of ANRIL in osteosarcoma remains unknown.

In the present study, the expression of ANRIL was observed to be upregulated in osteosarcoma tissues when compared with ANCT, which suggests that ANRIL may serve an important role in osteosarcoma development and progression. To investigate the underlying mechanisms of the ANRIL IncRNA in osteosarcoma progression further, the expression of ANRIL was reduced in U2OS osteosarcoma cells by lentivirus-mediated RNA interference. RT-qPCR analysis was subsequently performed to determine the expression of ANRIL in U2OS osteosarcoma cells following transfection with si-ANRIL or si-NC. MTT, colony formation and transwell assays were used to analyze the proliferation, migration and invasion capacity of transfected U2OS osteosarcoma cells. The results of the current study demonstrated that ANRIL knockdown significantly decreased the proliferation, migration and invasion of osteosarcoma cells in vitro. This indicates the importance of ANRIL in the cellular biology and oncogenesis of osteosarcoma cells.

In conclusion, ANRIL was observed to be upregulated in human osteosarcoma tissues. In addition, knockdown of ANRIL reduced osteosarcoma cell proliferation, invasion and migration in vitro. The current study identified ANRIL expression as a potential novel diagnostic marker and therapeutic target of osteosarcoma; however, further study is required in order to identify the exact pathway through which ANRIL influences osteosarcoma, and how this pathway works.

\section{Acknowledgements}

The current study was supported by the Natural Science Foundation of Science and Technology Department of Liaoning Province (grant no. 201302106) and the Science and Technology Department of Shenyang City (grant no. F14-231-1-48).

\section{References}

1. Huang J, Ni J, Liu K, Yu Y, Xie M, Kang R, Vernon P, Cao L and Tang D: HMGB1 promotes drug resistance in osteosarcoma. Cancer Res 72: 230-238, 2012.

2. Clark JC, Dass CR and Choong PF: A review of clinical and molecular prognostic factors in osteosarcoma. J Cancer Res Clin Oncol 134: 281-297, 2008.

3. Wilusz JE, Sunwoo H and Spector DL: Long noncoding RNAs: Functional surprises from the RNA world. Genes Dev 23: 1494-1504, 2009.

4. Mercer TR, Dinger ME and Mattick JS: Long non-coding RNAs: Insights into functions. Nat Rev Genet 10: 155-159, 2009.

5. Ulitsky I and Bartel DP: lincRNAs: Genomics, evolution, and mechanisms. Cell 154: 26-46, 2013.

6. Ginger MR, Shore AN, Contreras A, Rijnkels M, Miller J, Gonzalez-Rimbau MF and Rosen JM: A noncoding RNA is a potential marker of cell fate during mammary gland development. Proc Natl Acad Sci USA 103: 5781-5786, 2006.

7. Geisler S and Coller J: RNA in unexpected places: Long non-coding RNA functions in diverse cellular contexts. Nat Rev Mol Cell Biol 14: 699-712, 2013.

8. Yacqub-Usman K, Pickard MR and Williams GT: Reciprocal regulation of GAS5 lncRNA levels and mTOR inhibitor action in prostate cancer cells. Prostate 75: 693-705, 2015. 
9. Xu TP, Huang MD, Xia R, Liu XX, Sun M, Yin L, Chen WM, Han L, Zhang EB, Kong R, et al: Decreased expression of the long non-coding RNA FENDRR is associated with poor prognosis in gastric cancer and FENDRR regulates gastric cancer cell metastasis by affecting fibronectin1 expression. J Hematol Oncol 7: 63, 2014.

10. Yap KL, Li S, Muñoz-Cabello AM, Raguz S, Zeng L, Mujtaba S, Gil J, Walsh MJ and Zhou MM: Molecular interplay of the noncoding RNA ANRIL and methylated histone H3 lysine 27 by polycomb CBX7 in transcriptional silencing of INK4a. Mol Cell 38: 662-674, 2010.

11. Pasmant E, Laurendeau I, Heron D, Vidaud M, Vidaud D and Bieche I: Characterization of a germ-line deletion, including the entire INK4/ARF locus, in a melanoma-neural system tumor family: Identification of ANRIL, an antisense noncoding RNA whose expression coclusters with ARF. Cancer Res 67: 3963-3969, 2007.

12. Kotake Y, Nakagawa T, Kitagawa K, Suzuki S, Liu N, Kitagawa M and Xiong Y: Long non-coding RNA ANRIL is required for the PRC2 recruitment to and silencing of p15(INK4B) tumor suppressor gene. Oncogene 30: 1956-1962, 2011.

13. Aguilo F, Zhou MM and Walsh MJ: Long noncoding RNA, polycomb, and the ghosts haunting INK4b-ARF-INK4a expression. Cancer Res 71: 5365-5369, 2011.

14. Zhang EB, Kong R, Yin DD, You LH, Sun M, Han L, Xu TP, Xia R, Yang JS, De W and Chen Jf: Long noncoding RNA ANRIL indicates a poor prognosis of gastric cancer and promotes tumor growth by epigenetically silencing of miR-99a/miR-449a. Oncotarget 5: 2276-2292, 2014.

15. Huang MD, Chen WM, Qi FZ, Xia R, Sun M, Xu TP, Yin L, Zhang EB, De W and Shu YQ: Long non-coding RNA ANRIL is upregulated in hepatocellular carcinoma and regulates cell apoptosis by epigenetic silencing of KLF2. J Hematol Oncol 8: $50,2015$.
16. Livak KJ and Schmittgen TD: Analysis of relative gene expression data using real-time quantitative PCR and the 2(-Delta Delta C(T)) method. Methods 25: 402-408, 2001.

17. Ponting CP, Oliver PL and Reik W: Evolution and functions of long noncoding RNAs. Cell 136: 629-641, 2009.

18. Tsai MC, Manor O, Wan Y, Mosammaparast N, Wang JK, Lan F, Shi Y, Segal E and Chang HY: Long noncoding RNA as modular scaffold of histone modification complexes. Science 329: 689-693, 2010.

19. Wapinski O and Chang HY: Long noncoding RNAs and human disease. Trends Cell Biol 21: 354-361,2011.

20. Gupta RA, Shah N, Wang KC, Kim J, Horlings HM, Wong DJ, Tsai MC, Hung T, Argani P, Rinn JL, et al: Long non-coding RNA HOTAIR reprograms chromatin state to promote cancer metastasis. Nature 464: 1071-1076, 2010.

21. Wang CM, Wu QQ, Li SQ, Chen FJ, Tuo L, Xie HW, Tong YS, Ji L, Zhou GZ, Cao G, et al: Upregulation of the long non-coding RNA PlncRNA-1 promotes esophageal squamous carcinoma cell proliferation and correlates with advanced clinical stage. Dig Dis Sci 59: 591-597, 2014.

22. Ma MZ, Li CX, Zhang Y, Weng MZ,Zhang MD, Qin YY, Gong W and Quan ZW: Long non-coding RNA HOTAIR, a c-Myc activated driver of malignancy, negatively regulates miRNA-130a in gallbladder cancer. Mol Cancer 13: 156, 2014.

23. Barsotti AM, Beckerman R, Laptenko O, Huppi K, Caplen NJ and Prives C: p53-Dependent induction of PVT1 and miR-1204. J Biol Chem 287: 2509-2519, 2012

24. Lin L, Gu ZT, Chen WH and Cao KJ: Increased expression of the long non-coding RNA ANRIL promotes lung cancer cell metastasis and correlates with poor prognosis. Diagn Pathol 10: 14, 2015. 